\title{
Preparation and Stability of Inorganic Solidified Foam for Preventing Coal Fires
}

\author{
Botao Qin, ${ }^{1,2}$ Yi Lu, ${ }^{2}$ Fanglei Li, ${ }^{2}$ Yuwei Jia, ${ }^{2}$ Chao Zhu, ${ }^{2}$ and Quanlin Shi ${ }^{2}$ \\ ${ }^{1}$ State Key Laboratory of Coal Resources and Mine Safety, CUMT, Xuzhou, Jiangsu 221008, China \\ ${ }^{2}$ Faculty of Safety Engineering, China University of Mining and Technology, Xuzhou, Jiangsu 221116, China \\ Correspondence should be addressed to Botao Qin; qbt2003@163.com
}

Received 14 March 2014; Revised 21 May 2014; Accepted 26 May 2014; Published 2 July 2014

Academic Editor: Peter Chang

Copyright (c) 2014 Botao Qin et al. This is an open access article distributed under the Creative Commons Attribution License, which permits unrestricted use, distribution, and reproduction in any medium, provided the original work is properly cited.

\begin{abstract}
Inorganic solidified foam (ISF) is a novel material for preventing coal fires. This paper presents the preparation process and working principle of main installations. Besides, aqueous foam with expansion ratio of 28 and 30 min drainage rate of $13 \%$ was prepared. Stability of foam fluid was studied in terms of stability coefficient, by varying water-slurry ratio, fly ash replacement ratio of cement, and aqueous foam volume alternatively. Light microscope was utilized to analyze the dynamic change of bubble wall of foam fluid and stability principle was proposed. In order to further enhance the stability of ISF, different dosage of calcium fluoroaluminate was added to ISF specimens whose stability coefficient was tested and change of hydration products was detected by scanning electron microscope (SEM). The outcomes indicated that calcium fluoroaluminate could enhance the stability coefficient of ISF and compact hydration products formed in cell wall of ISF; naturally, the stability principle of ISF was proved right. Based on above-mentioned experimental contents, ISF with stability coefficient of $95 \%$ and foam expansion ratio of 5 was prepared, which could sufficiently satisfy field process requirements on plugging air leakage and thermal insulation.
\end{abstract}

\section{Introduction}

Coal fires are difficult, persistent, and costly problems worldwide in coal mining processes $[1,2]$. They lead to serious environmental issues, safety problems, and considerable economic losses [3]. Meanwhile, spontaneous combustion of coal and heat transfer occurs more frequently due to subsidence and increased channels of air leakage in the goaf. Air leakage prevention and thermal insulation can lower effectively the spontaneous combustion risk of coal $[4,5]$. Based on the various techniques for control and extinguishment of coal fires developed and applied over past 60 years [6-8], materials with pore structure are drawing growing attention because of their characteristics involving heat insulation, fire resistance, lightweight, superior fluidity, environmental friendliness, and so on $[9,10]$.

In this work, a novel material, ISF, with high closed porosity and uniform pore distribution was prepared via mixing aqueous foam and composite slurry consisting of fly ash, cement, and compound additives. In this process of preparation, the following two points are worthy of consideration. Firstly, stable aqueous foam is required for ISF to plug air leakage in mining applications. Furthermore, the stability of foam may be affected by foam generator parameters, surfactants, and their concentration [11]. Selection of surfactants has an impact on the properties of foam as it affects the surface tension and gas-liquid interfacial properties. Secondly, the foam slurry after mixing is a three-phase system (gasliquid-solid), which should be uniform and stable. But few scholars have studied the foam formation and stabilization in this kind of system. Current researches mainly focus on the stabilization of two-phase foam (aqueous foam or other liquid foam) [12]. Just a few scholars such as Gonzenbach et al. [13], Hunter et al. [14], Sethumadhavan et al. [15], and Vijayaraghavan et al. [16] carried out experimental studies and mechanism analyses on solid particles stabilized aqueous foam.

In this paper, as a first step, we studied the preparation process of ISF and analyzed the working principles and the effects of key devices. As a next step, the aqueous foam with 
low drainage rate and high expansion ratio was prepared based on sodium dodecyl sulfate (SDS) solution and modified by the foam stabilizers such as cetrimonium bromide $(\mathrm{CTAB})$, sodium chloride $(\mathrm{NaCl})$, and lauryl alcohol (LA). Then the factors influencing the stability coefficient and foam expansion ratio of ISF were investigated. At last, through the observation on drainage of the bubble wall and the hydration products accelerated by calcium fluoroaluminate, the stabilization mechanism of foam fluid was proposed.

\section{Experimental}

2.1. Raw Materials. Constituent materials are listed below.

(1) Portland cement (PC) with the compressive strength of $64.5 \mathrm{MPa}$ at 28 days, conforming to BSEN 197-1 type I cement [17].

(2) Fly ash (FA) with a median particle size of $35 \mu \mathrm{m}$ and loss on ignition (LOI) of $5.0 \%$, conforming to BS EN 450 [18].

(3) Calcium fluoroaluminate $\left(11 \mathrm{CaO} \cdot 7 \mathrm{Al}_{2} \mathrm{O}_{3} \cdot \mathrm{CaF}_{2}\right)$ : it influences the rate of cement hydration, leading to a reduction in setting time.

(4) Redispersible polymer powder (PP): it is a kind of polymeric powder which can be easily reemulsified in water to reform liquid emulsion with essentially identical properties to the original emulsion.

(5) Water (W): its percentage was fixed in order to satisfy both the workability criterion and the controlled low strength materials (CLSM) recommendations for the insulation materials [19].

(6) Lauryl sodium sulfate (SDS), Cetrimonium Bromide (CTAB), $\mathrm{NaCl}$, and lauryl alcohol (LA). They were diluted with water in different ratios.

2.2. Preparation Process of ISF. The basic preparation process can be divided into three parts including mixing the composite slurry, preparing aqueous foam, and mixing composite slurry, accelerator, and aqueous foam. We admixed cement, fly ash, and redispersible polymer powder together and got the blend of these three basic raw materials. Then, part of water was injected into the blend and composite slurry formed under the work of stirrer. The water-solid ratio was controlled slightly less than preset ratio. At the same time, the rest of the water was used to dilute the surfactant. Then, high pressure air was pumped into the foam generator and aqueous foam was produced. The next procedure was to mix composite slurry with aqueous foam in a self-made mixer, with some compound additives added. At last, foam fluid was produced and evolved into ISF at room temperature. The specific preparation procedures of ISF are schematically shown in Figure 1. The main installations are shown in Figure 2.

\subsection{Test Procedure}

2.3.1. Drainage Rate Test. We chose the drainage rate to be 30 minutes since aqueous foam was produced, to reflect foam stability. After generation of aqueous foam, the initial foam mass, $m$, was measured immediately and then poured fully into a Buchner funnel. A measuring cylinder was placed under the Buchner funnel, and the mass of liquid drained from aqueous foam, $m_{d}$, was calculated every ten minutes, until 30 minutes. Drainage rate, $d_{t}$, can be expressed by

$$
d_{t}=\frac{m_{d}}{m} \times 100 \%
$$

2.3.2. Stability Coefficient Test. The test instrument was one cylindrical gauge whose inner diameter was $100 \mathrm{~mm}$ and measuring range was $315 \mathrm{~mm}$. The test procedure is as follows: (i) pour the fresh ISF into the test instrument and record the initial height, $h_{0}$; (ii) measure the final height $\left(h_{i}\right)$ when ISF turns into solidification state. The stability coefficient, $\psi$, can be calculated according to the following:

$$
\psi=\frac{h_{i}}{h_{0}} \times 100 \% .
$$

2.3.3. Foam Expansion Ratio Test. Test procedure for foam expansion ratio of aqueous foam or foam fluid is as follows: (i) fill a container (volume and mass are known and designated by $V_{c}, m_{c}$, resp.) with surfactant solution or cement slurry, weigh the total mass, $m_{L}$, and calculate the density of surfactant solution or cement slurry, $\rho_{L}$, by (3); (ii) overfill the aforementioned container with aqueous foam or foam fluid and strike off the excess foam, weigh the total mass, $m_{F}$, and calculate the density of aqueous foam or foam fluid, $\rho_{F}$, by (4); (iii) calculate the foam expansion ratio of aqueous foam or foam fluid, $E_{F}$, by (5) as follows:

$$
\begin{gathered}
\rho_{L}=\frac{m_{L}-m_{c}}{V_{c}}, \\
\rho_{F}=\frac{m_{F}-m_{c}}{V_{c}}, \\
E_{F}=\frac{\rho_{L}}{\rho_{F}} .
\end{gathered}
$$

2.3.4. Microscopy Observation. The microstructure of aqueous foam and ISF fluid were observed by a Nomarski-type phase contrast interference microscope equipped with a digital camera, which can be used to take photomicrograph of the samples and the foams. A drop of sample was brought onto a microscope slide and the structure of bubble was observed. The bubble wall of ISF was investigated by scanning electron microscopy (SEM) (FEI QuantaTM 250 SEM system) with the size of test specimen being $10 \times 10 \times 10 \mathrm{~mm}^{3}$ prism.

2.3.5. Particle Contact Angle Measurement. The water and surfactant solution contact angle on the particles was measured using the gravimetric version of the Washburn method. The method is based on measuring the penetration rate of 


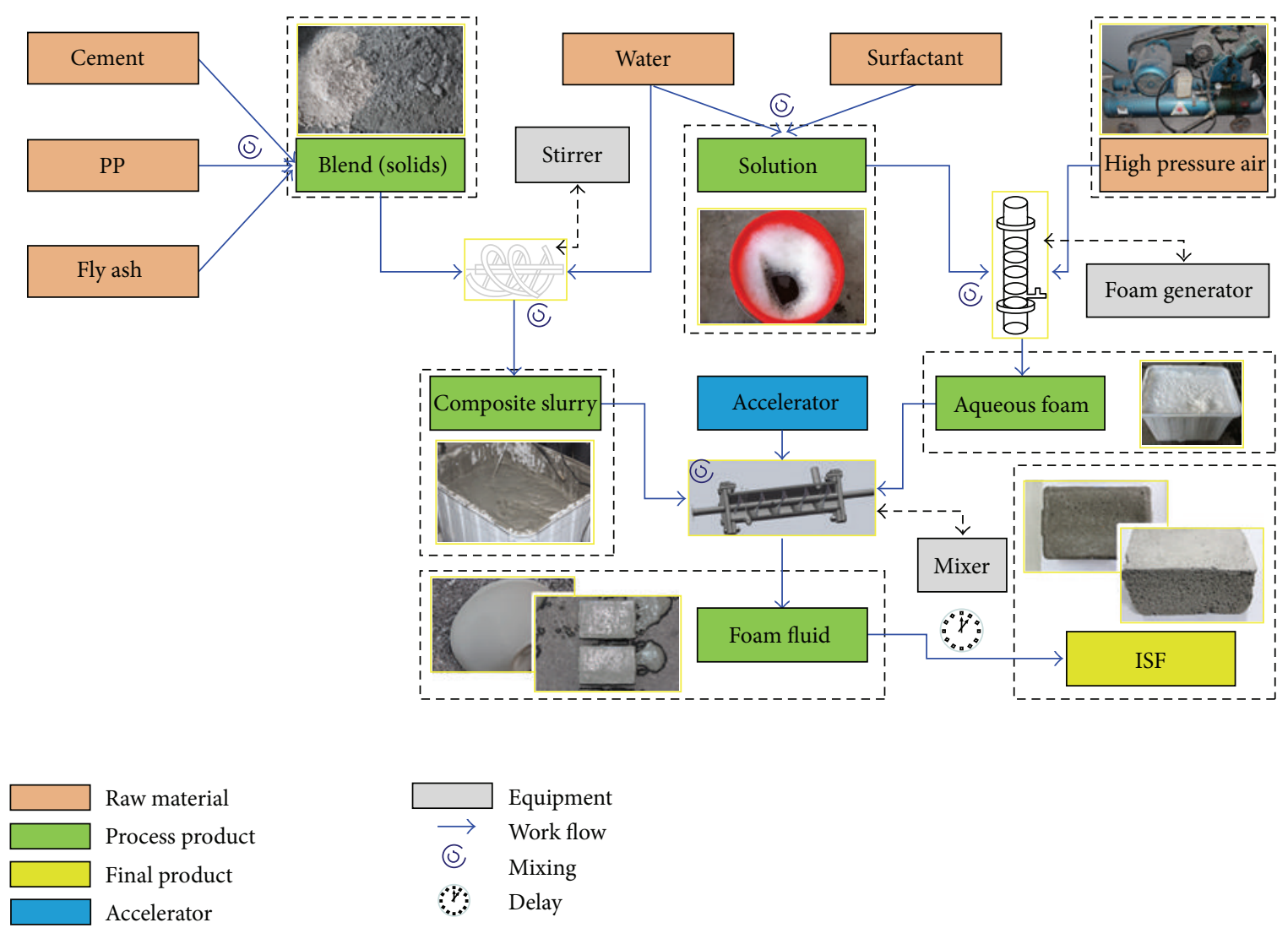

FIGURE 1: The schematic of the preparation of ISF.

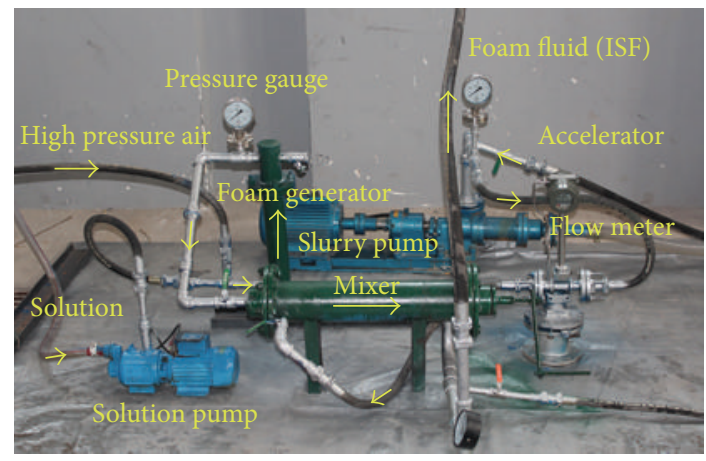

FIGURE 2: The main installations.

a wetting liquid into a packed bed of particles, which lead to the following equation [20]:

$$
M^{2}=t \times \frac{\gamma_{\mathrm{LG}} \delta^{2} \cos \theta}{\mu} \times \frac{r S^{2} \varepsilon^{2}}{2},
$$

where $M$ is the measured mass of the penetrated liquid; $t$ is the penetration time; $\gamma_{\mathrm{LG}}$ is the gas-liquid surface tension; $\delta$ is the liquid density; $S$ is the cross-sectional area of the tube; $\varepsilon$ is the void fraction of particles; $\mu$ is the viscosity of liquid; $r$ is the mean radius; $\theta$ is the contact angle.
2.4. Test Design. In order to investigate the influencing mechanism of aqueous foam volume (FV), fly ash replacement for cement (FA), and water-solid ratio (W/S) on the stability of ISF, we conducted tests on different specimens. FV was controlled to vary from $2 \mathrm{~V}$ to $10 \mathrm{~V}$ with the increment being $2 \mathrm{~V}$, FA changed as $10 \%, 20 \% . .50 \%$, and W/S increased from 0.3 to 0.5 with every difference quantity being 0.05 . According to this design, we conducted 125 tests.

\section{Results and Discussion}

3.1. The Key Devices. To develop fine, uniform, and stable inorganic solidified foam, the following two points deserve consideration. Firstly, the foam generator should be able to produce aqueous foam with uniform pore structure, high expansion ratio, and a certain stabilization time. Secondly, aqueous foam and composite slurry should contact thoroughly and then form stable foam fluid during the mixing process in the mixer. The schematic of key devices was shown in Figure 3.

The main process of generating foams by the homemade foam generator is as follows: once foaming agent solution and high pressure air flow through the T-shape conduit of foam generator, the turbulent eddy is formed after mixing and enhanced by the porous medium which can be composed of multilayer meshes, powdered metal, 


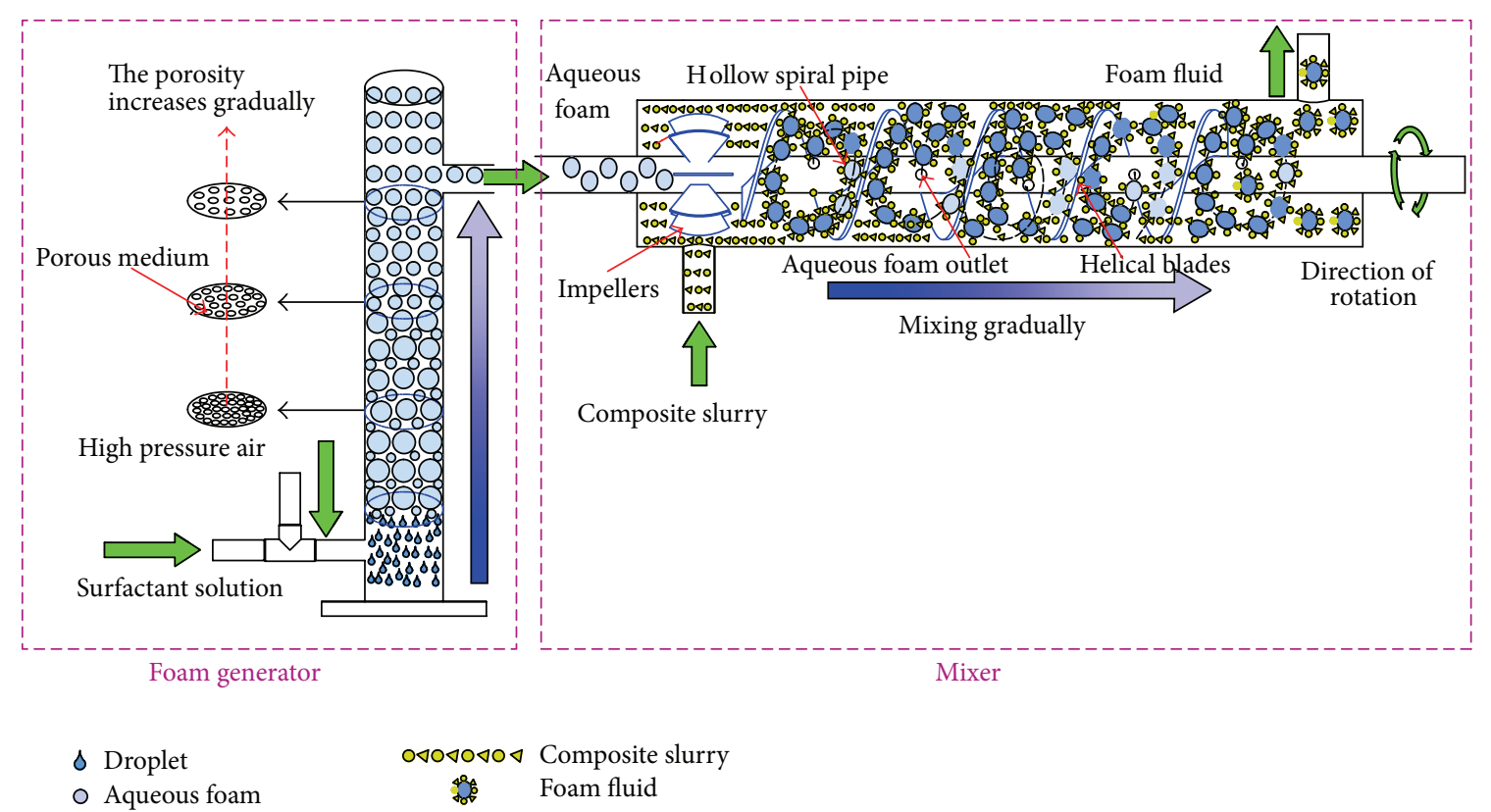

FIGURE 3: The schematic of foam generator and self-made mixer.

or spherical glass particles, causing greater pressure drop due to their impediment. The more homogenous and denser aqueous foam is produced from down to up as the porosity of porous medium increases stepwise. The aqueous foam produced by mechanical agitation and home-made foam generator was as shown in Figure 4.

Mixer consists of chamber and hollow spiral pipe inside it. The high-speed composite slurry drives the impellers to rotate, and then foam slurry is stirred and delivered by hollow spiral pipe with helical blades. Vortex streets in this process can completely go into turbulence and cause vortex according to certain frequency. The loss of kinetic energy acts on the mixtures and a large number of foam fluids are formed. Aqueous foams pass into the mixer from the left body of hollow spiral pipe equipped with five aqueous foam outlets with an interval angle. Aqueous foams are added to slurry step by step, which reduce the broken rate of foam and increase foam slurry contact areas. This kind of mixing chamber can weaken the shock caused by larger flow of aqueous foam and is conducive for gas-liquid-solid to mix thoroughly.

3.2. Preparation of Aqueous Foam. From viewing of the technology process for preparing the ISF, the stability is mainly dependent on that of aqueous foam. Generally speaking, foam expansion ratio of aqueous foam should be more than 20. SDS is a widely used surfactant with strong foaming ability. Its change trends of $30 \mathrm{~min}$ drainage rate and foam expansion ratio with different SDS concentrations are depicted in Figure 5.

From Figure 5, with increasing concentration of SDS, foam expansion ratio increases firstly and then decreases, for the reason that the surface tension of surfactant solution decreased firstly and then increased due to formation of surfactant micelle, and the largest foam expansion ratio is 24 under a concentration of 2.5\%. Drainage rate of aqueous foam presented a reverse trend compared to that of foam expansion ratio, whose minimum is $35 \%$ under a concentration of $2 \%$. This is for the reason that more micelles formed and their shape changed with the increase of SDS, contributing to more stable foam films and less drainage rate. However, in the other limit, that is, above $2 \%$, the violation of the law at higher micelle concentrations is related to the appearance of a freezing transition in foam films [21]. Considering the above two indexes, the optimal SDS concentration is $2.5 \%$.

In order to strengthen stability of aqueous foam, CTAB, $\mathrm{NaCl}$, and LA were utilized as foam stabilizers. We studied modification effects on SDS aqueous foam under different concentrations of foam stabilizers ranging from $0.5 \%$ to $4.0 \%$, whose concrete effects on foam expansion ratio and drainage rate are shown in Figure 6.

From Figure 6(a), the change trends of foam expansion ratio for three foam stabilizers are different, and with increasing concentration, that of $\mathrm{CTAB}$ declines and $\mathrm{NaCl}$ increases slightly, while LA elevates. In Figure 6(b), from the viewpoint of drainage rate, three foam stabilizers wholly could diminish the drainage rate of aqueous foam, specifically, with the increase of concentration; the drainage rate firstly falls off sharply and tardily goes up later. The minimums of drainage rate and the critical concentrations for $\mathrm{CTAB}, \mathrm{NaCl}$, and LA are $(20 \%, 2.5 \%),(26 \%, 1.0 \%)$, and $(13 \%, 2.0 \%)$, respectively. The reasons accounting for the trends mentioned above are special as follows.

Under the condition that the concentration of SDS is $2.5 \%$, its foam expansion ratio decreases with the increasing concentration of CTAB. Because CTAB is a cationic surfactant while SDS is an anionic one, when these two surfactants are mixed, phase separation will occur due to intense electrostatic interaction and condensation of surfactant molecules [22], followed by the ascent of surface tension. 


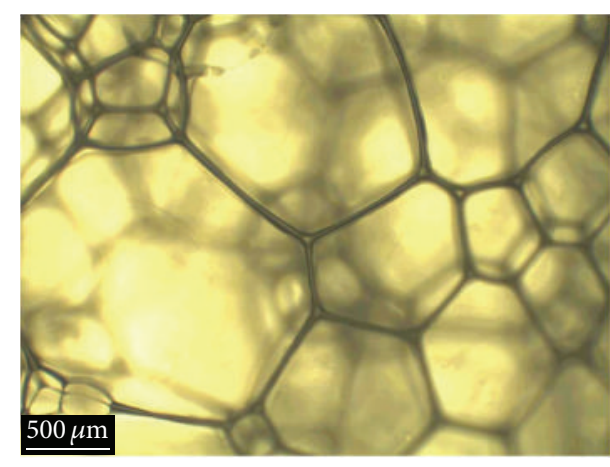

(a) Produced by mechanical agitation

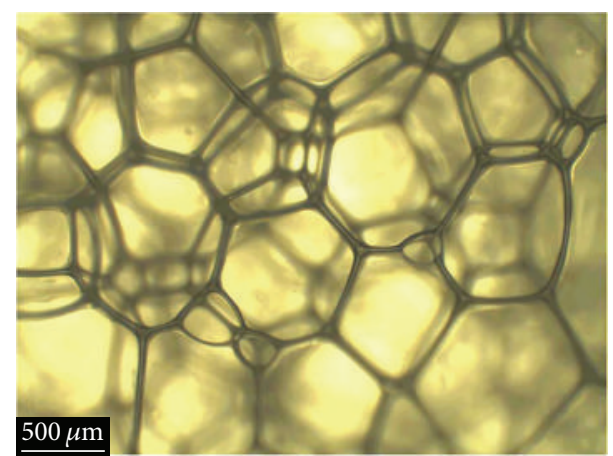

(b) Produced by the home-made foam generator

Figure 4: The optical microscopic analysis diagram of aqueous foam.

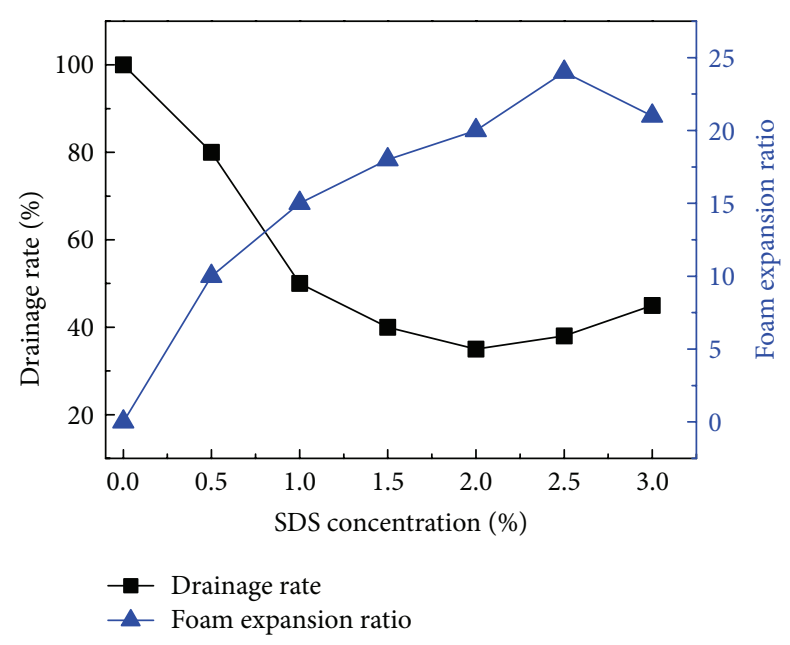

Figure 5: Change trends of drainage rate and foam expansion ratio with different concentrations.

With the increase of $\mathrm{CTAB}$ concentration, the drainage rate of aqueous foam decreases firstly and then increases, which is $20 \%$ and the least under a concentration of $2.5 \%$. Compared with the individual SDS system, the SDS+CTAB mixed system had a synergic effect on foam stabilization [23]. Surfactant mixtures could create a mixed surfactant layer at gas/liquid interfaces. When two bubbles are approaching each other to form a thin liquid film, this mixed surfactant layer can confer disjoining pressures to hinder this approaching.

The foam expansion ratio enlarges with the increase of $\mathrm{NaCl}$ concentration mainly because homo-ion could not only diminish the Critical Micelle Concentration (CMC) of the surfactant but also reduce surface tension of the solution and develop its foaming ability. The drainage rate decreases firstly and then ascends with the increase of $\mathrm{NaCl}$ concentration, the minimum of which is $26 \%$ at a concentration of $1.0 \%$. The addition of $\mathrm{NaCl}$ to SDS solution enlarged its foaming ability to some degree and reduced its drainage rate, which could be explained that there is a threshold of added electrolyte on stratification phenomenon of foam film, above which the phenomenon is not observed [24].
Based on our experimental results, we believe that $1.0 \%$ was just the threshold. Above $1.0 \%$ concentrations of $\mathrm{NaCl}$, bubbles ruptured asynchronously owing to different surface concentrations of $\mathrm{NaCl}$; thus the drainage rate of foam rose slightly with the increased concentrations of $\mathrm{NaCl}$.

The addition of LA could both prominently improve the foam expansion and greatly enhance the stability of aqueous foam. This is because the iceberg structure (a perfectly ordered structure formed by the LA molecules and water molecules) around the hydrocarbon chain in the alcohol makes it a spontaneous process for the alcohol to participate in the formation of micelle and thus bubble films are consolidated. The drainage rate of foam film will slow down with the rise of surfactant micelles in certain range of concentrations [25].

The prepared foam-forming solution containing SDS concentration of $2.5 \%$ and LA concentration of $2 \%$ possesses excellent foam expansion ratio with the value being 28 and the aqueous foam derived from the solution acquires the best stability with the value being $13 \%$.

\subsection{The Stability of ISF}

3.3.1. Aqueous Foam Volume, Fly Ash Replacement for Cement and Water-Solid Ratio. According to the results of 125 tests, it can be concluded that when FV is $8 \mathrm{~V}, \mathrm{FA}$ is $30 \%$ and W/S is 0.4 , and the ISF is in the best state with its foam expansion ratio and stability coefficient being $5 \mathrm{~V}$ and $90 \%$, respectively. At the same time, some other test data was shown in Figure 7.

From Figure 7(a), it can be seen that when FV increased, foam expansion ratio and stability coefficient of ISF show different variation trends. As the aqueous foam volume increases, the slurry system becomes more disperse and the film becomes thinner, which lead to the bursting of liquid film even if the drainage volume is not big. Besides, cement and fly ash particles cannot form a continuum and the setting and the hydration are slowed down. So, foam stability decreases as a function of increasing aqueous foam volume. When ISF is used in field, ISF is required with high foam expansion ratio and desired stability coefficient. But, in fact, these two targets cannot be achieved simultaneously. Therefore, we expect that, under the limit of foam expansion ratio which is not less than 


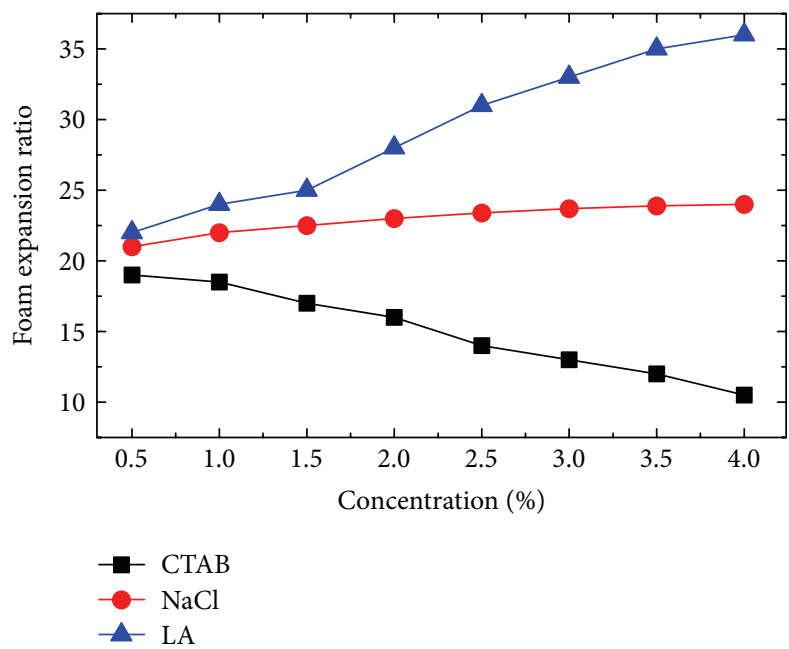

(a)

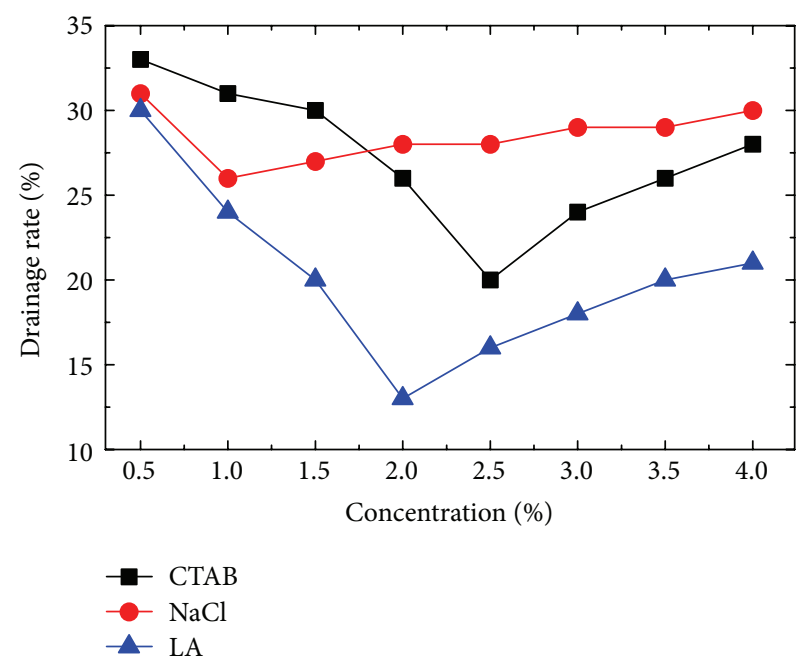

(b)

FIGURE 6: Modifying effects of foam stabilizer on drainage rate and foam expansion ratio.

$4 \mathrm{~V}$ according to the technological requirements, the stability coefficient should be improved as high as possible.

To reduce cost, we use a small quantity of fly ash to replace cement. Figure 7(b) shows the variation in foam expansion ratio and stability coefficient with FA. The foam expansion ratio and stability coefficient both reach the maximums when the FA is $30 \%$. This phenomenon can be explained as follows. When the cement particles are irregular geometry, there are many spherical particles (glass beads) in fly ash. Glass beads function like ball bearing reducing the friction among cement particles and increasing the liquidity of foam slurry, thus making bubbles disperse evenly. But the hydration velocity of fly ash is slower than cement. If the fly ash replacement level is too high, it can cause reduction of the early hydration products and rupture of bubbles and reduce the stability coefficient of ISF.

It is observed from Figure 7(c) that, with the increase of water cement ratio, foam expansion ratio and stability coefficient exhibit the same change trend. A possible reason for this is that, at a too low W/S level, cement hydration consumes the water of foam, leading to bubble rupture and foam slurry instability. However, when the W/S is too large, solid particles may sink and foam can float upward, which causes the uneven component of foam slurry and affects the stability of ISF.

3.3.2. The Enhancement of the Stability. The maximum stability coefficient is $90 \%$ based on the results of 125 groups of experiments. There are certain changes in its internal structure of foam fluid during the solidification from the fresh state. For a more in-depth study on the changes in the internal structure of the bubble, the fresh state of foam fluid (Figure 8) was observed by optical microscopic system.

Figure 8 shows that there are two distinct cases with respect to the cement particles' location. Most of the particles are present only inside the film and just a few particles are firmly attached to the film surface. In the first case, solid particles at sufficiently high concentration can form a layered structure inside the thinning film and thus stabilize it by the so called oscillatory structural force. In the second case, a few particles irreversibly adsorb at the gas-liquid interface and significantly increase the interfacial elasticity needed to prevent the film rupture and bubble coalescence. The foam stability has been quantitatively assessed by the particle hydrophobicity measured in terms of the contact angle, $\theta$, which is related with the energy, $G$, required to remove the small particles (radius being $R_{s}$ ) from the interface by the following [26]:

$$
G=\pi R_{s}^{2} \gamma_{\mathrm{LG}}(1-\cos \theta)^{2} .
$$

According to Binks, the optimum contact angle for foam stabilization is about $90^{\circ}$, as at this value the energy to remove the particle from the interface has the highest value. Experimentally, the optimum contact angle interval, ensuring the highest foam stability, was found between 40 and $70^{\circ}$ [27] and 75 and $85^{\circ}$ [28] (see also results in [29]). Based on (6), our measurements give a contact angle of $166^{\circ}$ and $78^{\circ}$ for the particles in water and surfactant solution, respectively. Therefore, the aqueous foams can be stabilized by solid particles. The adsorption of CTAB and LA molecules on the surfaces of the particles changes their hydrophobicity. The partially hydrophobic particles are able to attach to the interfaces, which play a crucial role in the high foam stability reported here [30]. For further investigation, the bursting process of an unstable bubble was shown in Figure 9.

From Figure 9(a) to Figure 9(b), this phenomenon was called limited coalescence and was observed with emulsion stabilized by the same type of particles [31]. After a drainage period the site where the liquid drained is clear (as compared with the dispersions which are turbid), and the foam evolves 


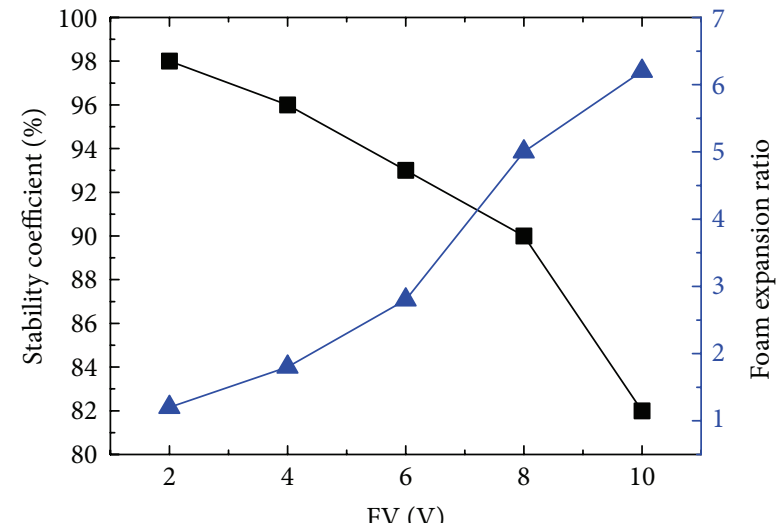

Stability coefficient Foam expansion ratio

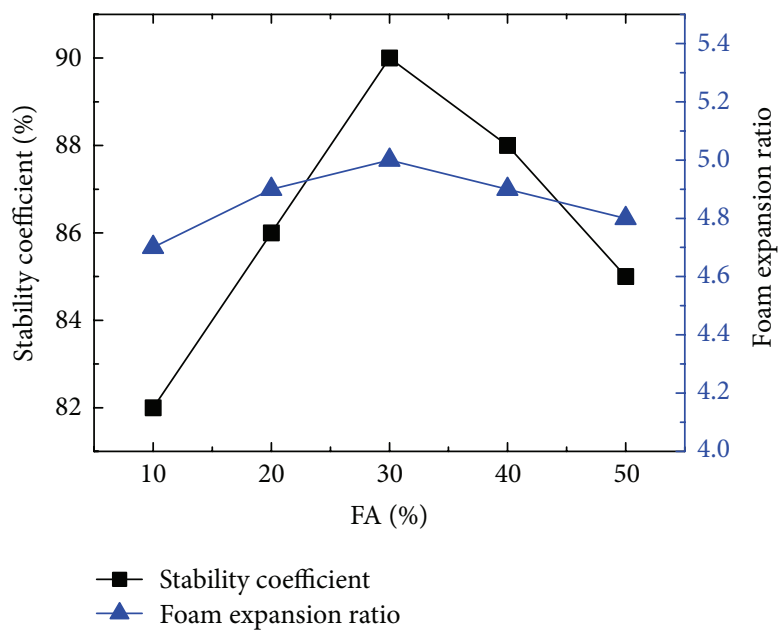

(b)

(a)

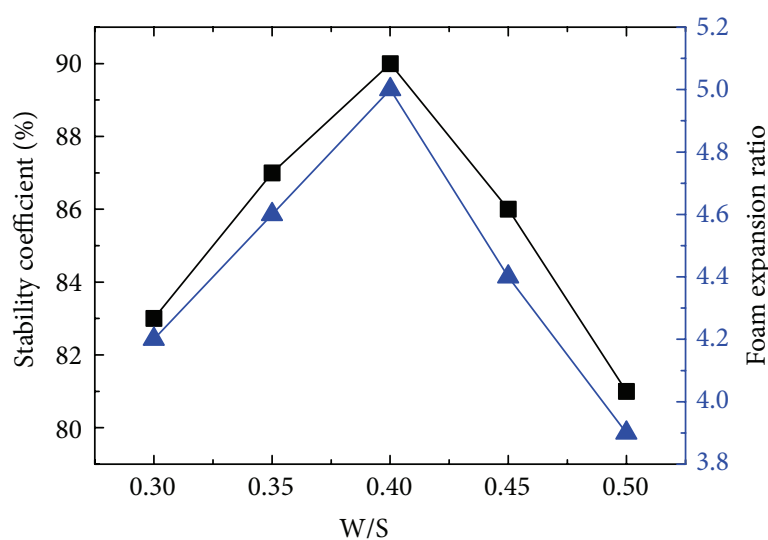

- Stability coefficient

Foam expansion ratio

(c)

Figure 7: The change curves of stability coefficient and foam expansion ratio with different factors. (a) Independent variable is FV and constants are $30 \mathrm{wt} . \%$ of FA and W/S of 0.4; (b) Independent variable is FA and constants are $8 \mathrm{~V}$ of FV and W/S of 0.4; (c) independent variable is $\mathrm{W} / \mathrm{S}$ and constants are $8 \mathrm{~V}$ of $\mathrm{FV}$ and $30 \mathrm{wt} . \%$ of FA.

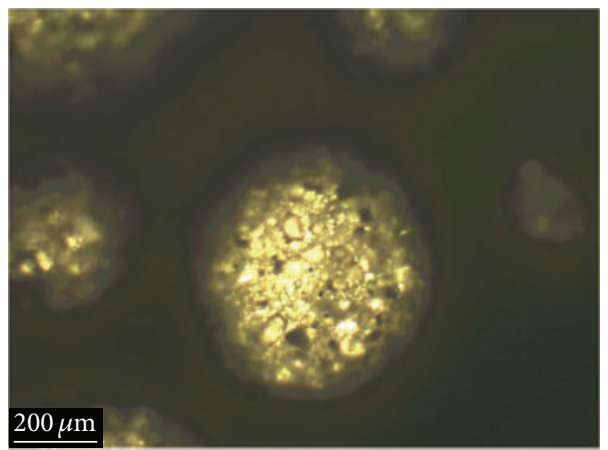

Figure 8: The fresh state of foam fluid. 


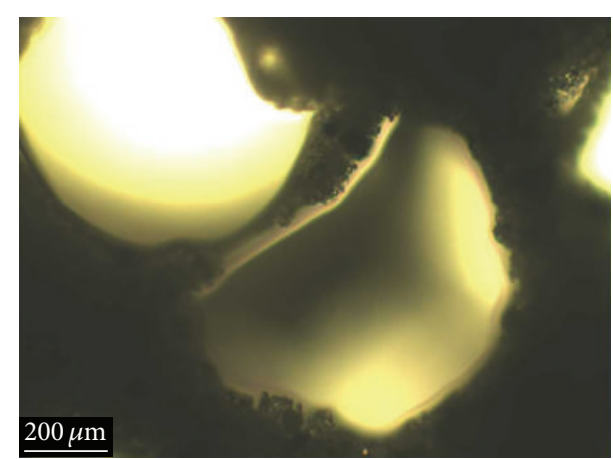

(a)

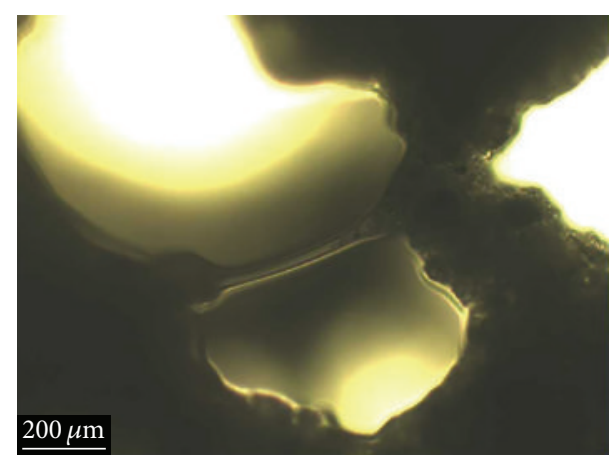

(b)

FIgURE 9: The bursting process of an unstable bubble.

little with time. If initially after creation the bubble surfaces are not sufficiently covered by particles, upon coalescence, the surface to volume ratio of the created bubbles decreases and hence eventually the coalescence proceeds $[32,33]$.

Based on the previous analysis, the apparent high stability against disproportionation is the most significant result, even considering the coagulated nature of the particles. Also, as with foam fluid, partial coagulation of particle networks on the surfaces of the bubbles is found to be advantageous for stability. It should be noted that the rate of drainage from the bubble wall is much faster than the rate of precipitation of the hydration products. So promoting the formation of hydration products is the correct way to delay and stop the burst of bubbles.

3.3.3. The Dynamic Changes of Bubble Wall after Adding Calcium Fluoroaluminate. Accelerators influence the rate of cement hydration, leading to particles with a high degree of internetworking against disproportionation and to occurrence of greater retardation. So we conduct experiments on the concentration of calcium fluoroaluminate $(11 \mathrm{CaO}$. $7 \mathrm{Al}_{2} \mathrm{O}_{3} \cdot \mathrm{CaF}_{2}$ ) on the foam stability coefficient as shown in Figure 10. Foam stability first increases and then decreases with the content increase of $11 \mathrm{CaO} \cdot 7 \mathrm{Al}_{2} \mathrm{O}_{3} \cdot \mathrm{CaF}_{2}$ and the maximum stability is $95 \%$ under the value of concentration being $12 \%$.

In cement-based materials (e.g., ISF), the transformation process from a paste phase into a solid phase can be understood from the properties of their constituents. When $11 \mathrm{CaO} \cdot 7 \mathrm{Al}_{2} \mathrm{O}_{3} \cdot \mathrm{CaF}_{2}$ is added to ISF system, $\mathrm{Al}_{2} \mathrm{O}_{3}$, coming from the admixture, could react with gypsum to form immediately ettringite crystals $\left(\left[\mathrm{Ca}_{2}(\mathrm{Al}, \mathrm{Fe})(\mathrm{OH})_{6}\right]_{2} \cdot \mathrm{X}_{3}\right.$. $n \mathrm{H}_{2} \mathrm{O}$ ), which will attach to the particle surface. At the same time, the consumption of gypsum accelerates the pace of tricalcium silicate $\left(3 \mathrm{CaO} \cdot \mathrm{SiO}_{2}\right)$ hydration, forming a small amount of fibrous $\mathrm{C}-\mathrm{S}-\mathrm{H}$ filling among the cement particles. The chemical reaction consists in the transformation of $11 \mathrm{CaO} \cdot 7 \mathrm{Al}_{2} \mathrm{O}_{3} \cdot \mathrm{CaF}_{2}$ into $\left[\mathrm{Ca}_{2}(\mathrm{Al}, \mathrm{Fe})(\mathrm{OH})_{6}\right]_{2} \cdot \mathrm{X}_{3} \cdot n \mathrm{H}_{2} \mathrm{O}$ via a dissolution precipitation process by (8). The dynamic

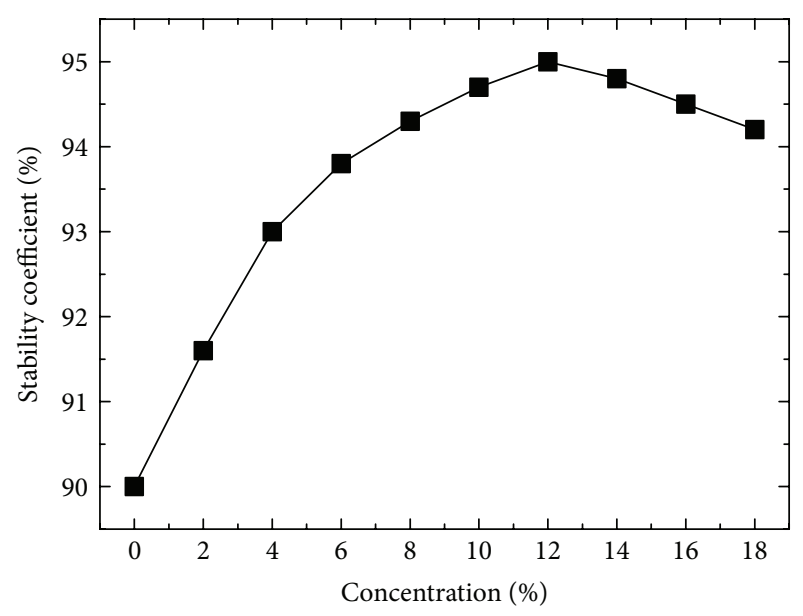

FIGURE 10: The foam stability coefficient versus concentration of accelerators.

changes of bubble wall in the stabilization and solidification process were shown in Figure 11. Consider

$$
\begin{aligned}
& 3\left(11 \mathrm{CaO} \cdot 7 \mathrm{Al}_{2} \mathrm{O}_{3} \cdot \mathrm{CaF}_{2}\right)+33 \mathrm{CaSO}_{4}+382 \mathrm{H}_{2} \mathrm{O} \\
& \longrightarrow \\
& \quad 11\left(3 \mathrm{CaO} \cdot \mathrm{Al}_{2} \mathrm{O}_{3} \cdot 3 \mathrm{CaSO}_{4} \cdot 32 \mathrm{H}_{2} \mathrm{O}\right) \\
& \quad+3 \mathrm{CaF}_{2}+10\left(\mathrm{Al}_{2} \mathrm{O}_{3} \cdot 3 \mathrm{H}_{2} \mathrm{O}\right)
\end{aligned}
$$

In the SEM image obtained from the sample after solidification, the evolution of the primary cement hydration products is obvious. We can observe the formation of ettringite as rod-like crystals massively fill capillary pores. Surface products such as $\mathrm{C}-\mathrm{S}-\mathrm{H}$ gel can be observed as the major ISF microstructure component. $\mathrm{CH}$ as a pore product with a polycrystalline shape is another dominant cement hydration product. The SEM shows that the cement and fly ash particles are more connected and cement hydration products completely surround the particles.

\section{Conclusions}

(1) This paper presents the manufacturing process of ISF which consists of mixing the composite slurry, 


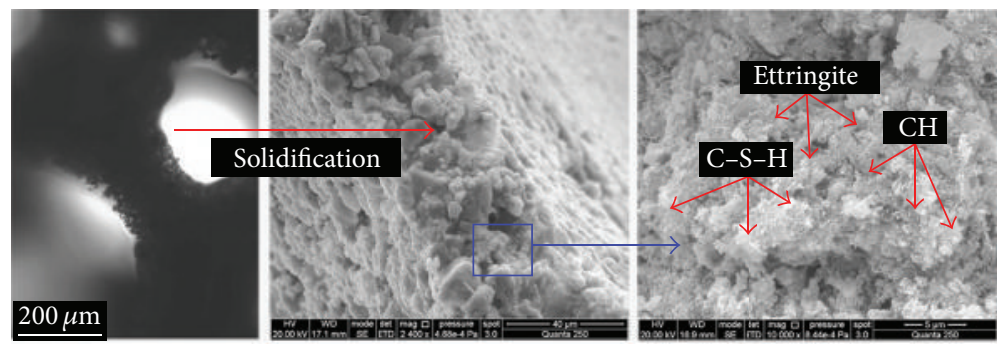

FIGURE 11: The SEM images of bubble wall.

preparing aqueous foam, and mixing them with accelerator. The foam generator can produce homogenous and dense aqueous foams due to the turbulent eddy which is formed and enhanced by the porous medium. A large number of foam fluids are formed by the self-made mixer in which turbulence and vortex were generated, and then aqueous foams were added stepwise to slurry.

(2) The aqueous foam with expansion ratio of 28 and 30 min drainage rate of $13 \%$ was obtained as a function of $2.5 \mathrm{wt} . \%$ SDS and $2 \mathrm{wt} . \%$ LA. The effects of FV, FA, and W/S on stability coefficient and foam expansion ratio were studied. And the results show that the optimum values of foam expansion ratio and stability coefficient were $5 \mathrm{~V}$ and $90 \%$, respectively, by value of FV being $8 \mathrm{~V}$, FA being 30\%, and W/S being 0.4 .

(3) The adsorption of CTAB and LA molecules on the surfaces of the particles changes their hydrophobicity with the contact angle from $166^{\circ}$ to $78^{\circ}$. The mechanism concerning accelerating the hydration and reducing the drainage was proposed and verified based on the analysis of dynamic change of bubble wall.

(4) At last, ISF with stability coefficient of $95 \%$ and foaming expansion ratio of 5 was fabricated, which could sufficiently satisfy field process requirements of air sealing and thermal insulation.

\section{Conflict of Interests}

The authors declare that there is no conflict of interests regarding the publication of this paper.

\section{Acknowledgments}

This work was supported by the National Natural Science Foundation of China (no. U1361213), the Fundamental Research Funds for the Central Universities (CUMT, 2014YC04), and the independent study projects of State Key Laboratory of Coal Resources and Mine Safety (SKLCRSM13X04).

\section{References}

[1] G. B. Stracher and T. P. Taylor, "Coal fires burning out of control around the world: Thermodynamic recipe for environmental catastrophe," International Journal of Coal Geology, vol. 59, no. 1-2, pp. 7-17, 2004.

[2] C. Kuenzer and G. B. Stracher, "Geomorphology of coal seam fires," Geomorphology, vol. 138, no. 1, pp. 209-222, 2012.

[3] M. A. Engle, L. F. Radke, E. L. Heffern et al., "Gas emissions, minerals, and tars associated with three coal fires, Powder River Basin, USA," Science of the Total Environment, vol. 420, pp. 146159, 2012.

[4] F. Zhou, B. Shi, Y. Liu, X. Song, J. Cheng, and S. Hu, "Coating material of air sealing in coal mine: clay composite slurry (CCS)," Applied Clay Science, vol. 80-81, pp. 299-304, 2013.

[5] B. Taraba, Z. Michale, V. Michalcová, T. Blejchař, M. Bojko, and M. Kozubková, "CFD simulations of the effect of wind on the spontaneous heating of coal stockpiles," Fuel, vol. 118, no. 2, pp. 107-112, 2014.

[6] T. R. Jolley and H. W. Russell, "Control of fires in inactivecoal deposits in Western United States, including Alaska, 19481958," Information Circular U.S. Bureau of Mines 7932, 1959.

[7] J. J. Feiler and G. J. Colaizzi, IHI Mine Fire Control Project Utilizing Foamed Grout Technology, Rifle, Colorado Bureau of Mines, United States Department of the Interior Research Contract Report 14320395H0002, 1996.

[8] F. B. Zhou, "Application of new material as air tight coating material in entries retained at gob-sides," Coal Safety Special Issue, pp. 97-98, 2009.

[9] A. Kan and H. Houde, "Effective thermal conductivity of open cell polyurethane foam based on the fractal theory," Advances in Materials Science and Engineering, vol. 2013, Article ID 125267, 7 pages, 2013.

[10] S. K. Lim, C. S. Tan, O. Y. Lim, and Y. L. Lee, "Fresh and hardened properties of lightweight foamed concrete with palm oil fuel ash as filler," Construction and Building Materials, vol. 46, pp. 39-47, 2013

[11] I. S. Ranjani and K. Ramamurthy, "Relative assessment of density and stability of foam produced with four synthetic surfactants," Materials and Structures, vol. 43, no. 10, pp. 1317$1325,2010$.

[12] E. Carey and C. Stubenrauch, "Free drainage of aqueous foams stabilized by mixtures of a non-ionic $\left(\mathrm{C}_{12} \mathrm{DMPO}\right)$ and an ionic (C12TAB) surfactant," Colloids and Surfaces A: Physicochemical and Engineering Aspects, vol. 419, pp. 7-14, 2013.

[13] U. T. Gonzenbach, A. R. Studart, E. Tervoort, and L. J. Gauckler, "Stabilization of foams with inorganic colloidal particles," Langmuir, vol. 22, no. 26, pp. 10983-10988, 2006. 
[14] T. N. Hunter, R. J. Pugh, G. V. Franks, and G. J. Jameson, “The role of particles in stabilising foams and emulsions," Advances in Colloid and Interface Science, vol. 137, no. 2, pp. 57-81, 2008.

[15] G. N. Sethumadhavan, A. D. Nikolov, and D. T. Wasan, "Stability of liquid films containing monodisperse colloidal particles," Journal of Colloid and Interface Science, vol. 240, no. 1, pp. 105112, 2001.

[16] K. Vijayaraghavan, A. Nikolov, and D. Wasan, "Foam formation and mitigation in a three-phase gas-liquid-particulate system," Advances in Colloid and Interface Science, vol. 123-126, pp. 4961, 2006.

[17] BS EN 197-1, Cement, Composition, Specifications and Conformity Criteria for Common Cements, British Standards Institution, London, UK, 1995.

[18] BS EN 450, Fly Ash for Concrete: Definitions, Requirements and Quality Control, British Standards Institution, London, UK, 1995.

[19] W. E. Brewer, Durability Factors Affecting CLSM. SP 150-3, American Concrete Institute, Detroit, Mich, USA, 1994.

[20] A. V. Nguyen, "Flotation," in Encyclopedia of Separation Science, I. D. Wilson, Ed., pp. 1-27, Elsevier, Amsterdam, The Netherlands, 2007.

[21] S. Grandner and S. H. Klapp, "Surface charge induced freezing of colloidal suspensions," Europhysics Letters, vol. 90, no. 6, Article ID 68004, 2010.

[22] S. I. Karakashev, E. D. Manev, R. Tsekov, and A. V. Nguyen, "Effect of ionic surfactants on drainage and equilibrium thickness of emulsion films," Journal of Colloid and Interface Science, vol. 318, no. 2, pp. 358-364, 2008.

[23] M. Wang, H. Du, A. Guo, R. Hao, and Z. Hou, "Microstructure control in ceramic foams via mixed cationic/anionic surfactant," Materials Letters, vol. 88, pp. 97-100, 2012.

[24] A. D. Nikolov and D. T. Wasan, "Ordered micelle structuring in thin films formed from anionic surfactant solutions. I. Experimental," Journal of Colloid And Interface Science, vol. 133, no. 1, pp. 1-12, 1989.

[25] S. E. Anachkov, K. D. Danov, E. S. Basheva, P. A. Kralchevsky, and K. P. Ananthapadmanabhan, "Determination of the aggregation number and charge of ionic surfactant micelles from the stepwise thinning of foam films," Advances in Colloid and Interface Science, vol. 183-184, pp. 55-67, 2012.

[26] B. P. Binks, "Particles as surfactants-similarities and differences," Current Opinion in Colloid and Interface Science, vol. 7, no. 1-2, pp. 21-41, 2002.

[27] G. Johansson and R. J. Pugh, "The influence of particle size and hydrophobicity on the stability of mineralized froths," International Journal of Mineral Processing, vol. 34, no. 1-2, pp. $1-21,1992$.

[28] Y. Q. Sun and T. Gao, "The optimum wetting angle for the stabilization of liquid-metal foams by ceramic particles: experimental simulations," Metallurgical and Materials Transactions A, vol. 33, no. 10, pp. 3285-3292, 2002.

[29] S. W. Ip, Y. Wang, and J. M. Toguri, "Aluminum foam stabilization by solid particles," Canadian Metallurgical Quarterly, vol. 38, no. 1, pp. 81-92, 1999.

[30] Q. Liu, S. Zhang, D. Sun, and J. Xu, "Aqueous foams stabilized by hexylamine-modified Laponite particles," Colloids and Surfaces A Physicochemical and Engineering Aspects, vol. 338, no. 1-3, pp. 40-46, 2009.

[31] E. Rio, W. Drenckhan, A. Salonen, and D. Langevin, "Unusually stable liquid foams," Advances in Colloid and Interface Science, vol. 205, pp. 74-86, 2014.
[32] S. Samanta and P. Ghosh, "Coalescence of bubbles and stability of foams in aqueous solutions of Tween surfactants," Chemical Engineering Research and Design, vol. 89, no. 11, pp. 2344-2355, 2011.

[33] W. Kracht and H. Rebolledo, "Study of the local critical coalescence concentration (1-CCC) of alcohols and salts at bubble formation in two-phase systems," Minerals Engineering, vol. 5051, pp. 77-82, 2013. 

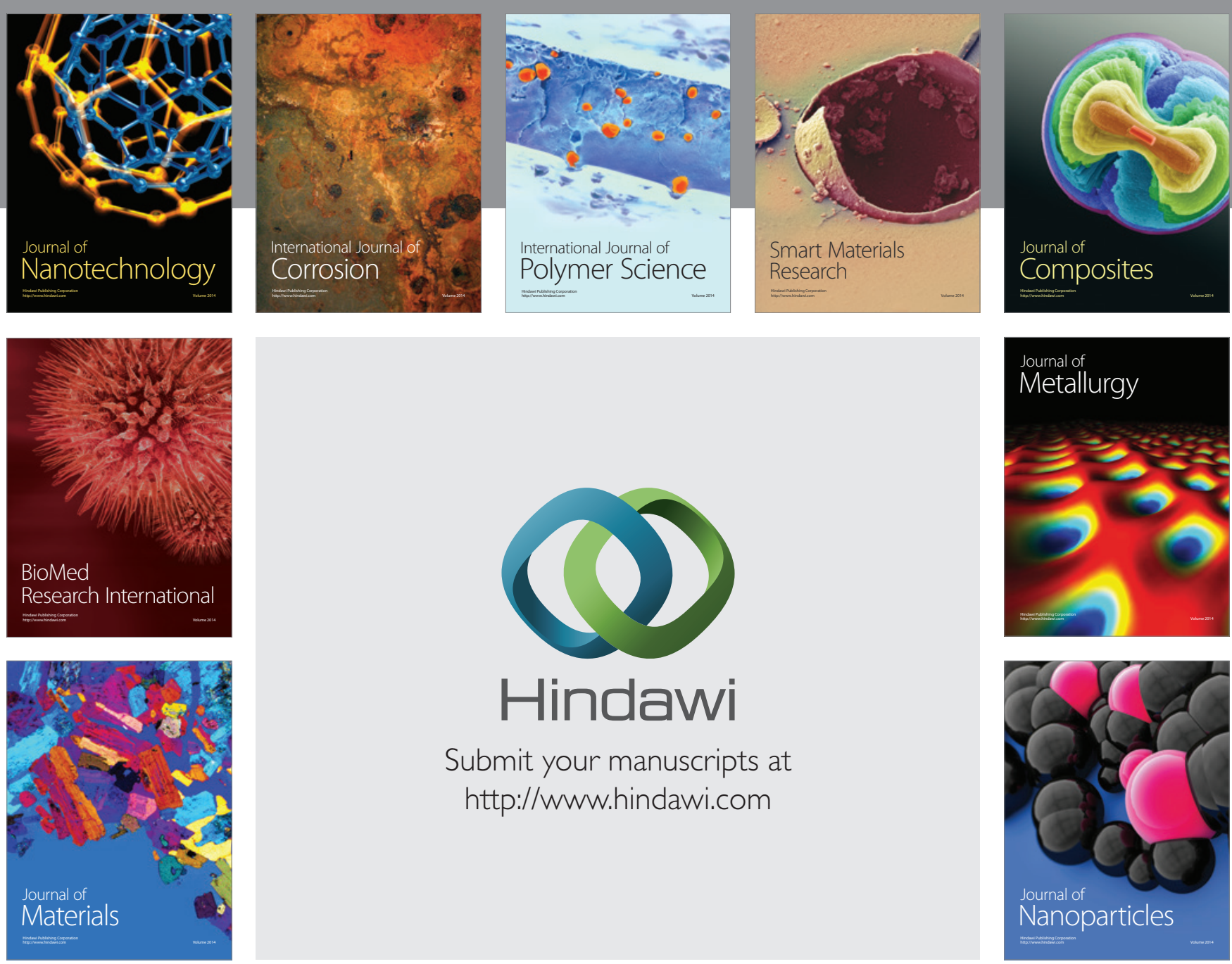

Submit your manuscripts at http://www.hindawi.com
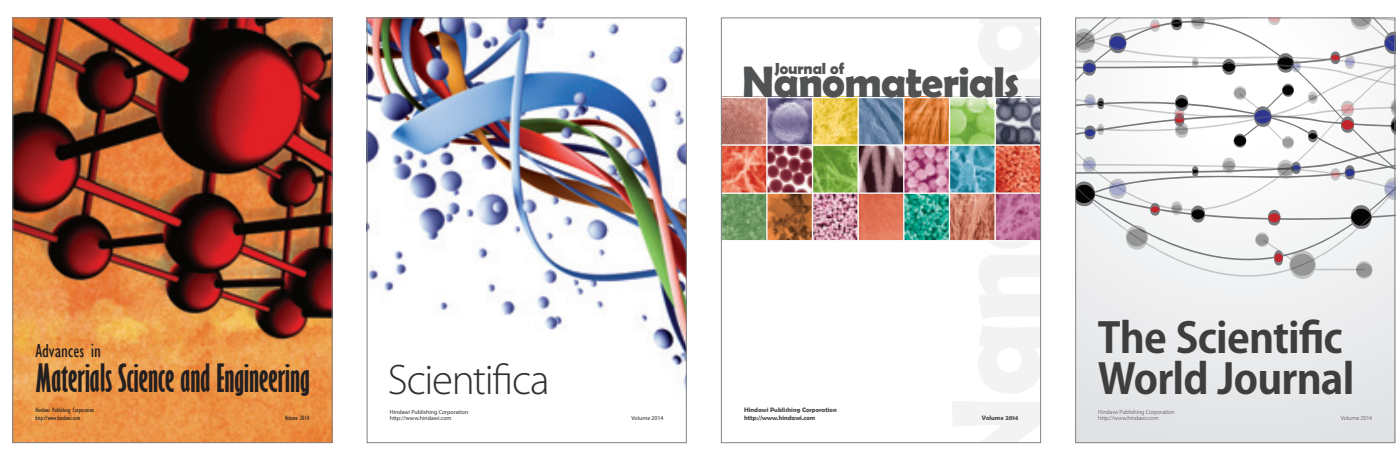

\section{The Scientific World Journal}
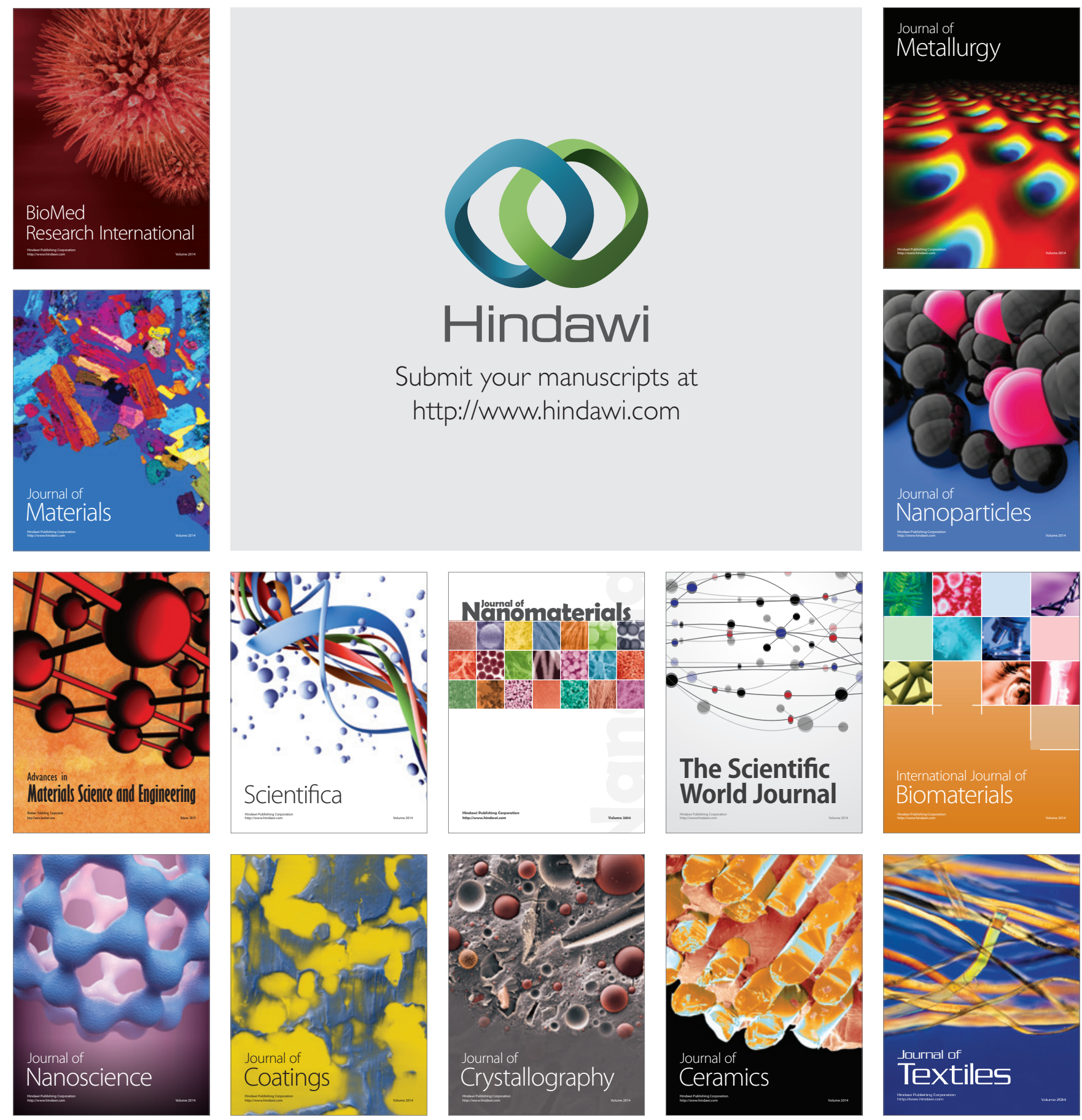